socioeconomic status were included at the patient level, and we explored a continuous outcome. We included both binary and continuous effects at the provider level, to reflect organisational features such as surgeon speciality or available beds, although these were analysed separately to demonstrate proofof-principle. We simulated unique sets of 100 datasets using a range of coefficient effect values and error variances. Interest lies in the ability of the MLC model to recover these simulated provider-level coefficient effects.

Results Models contained one patient-level latent class and up to five provider-level latent classes. For the binary providerlevel covariate, median recovered values were almost identical to simulated effects throughout, e.g. for the simulated coefficient value 0.500 at $33 \%$ error variance, the median recovered value was 0.499 (95\% CI 0.489-0.509) across all models. For the continuous provider-level covariate, median recovered values improved as the number of provider-level latent classes were increased, e.g. for the simulated coefficient value 0.200 at $33 \%$ error variance, the median recovered value was $0.153(95 \%$ CI $0.113-0.184)$ for two provider-level classes and 0.191 (95\% CI $0.168-0.210)$ for five providerlevel classes.

Discussion The MLC modelling approach achieved successful recovery of simulated coefficient values, within credible intervals for at least three provider-level latent classes. Very small simulated coefficient values were not recovered as well as higher values, which may be due to the variability introduced during simulation dominating the coefficient effect. There is also some attenuation of effect seen for the continuous provider-level covariate. We have demonstrated the utility of this approach to separate modelling for prediction (to accommodate patient casemix) and for causal inference (to explore provider-level effects) across a data hierarchy. There is much scope to extend the assessment of upper-level causal effects by consideration of a multivariable DAG.

\section{P19 BIRTH, CHILD AND MATERNAL OUTCOMES FOR YOUNG AND VULNERABLE MOTHERS IN ENGLAND: A POPULATION-BASED DATA LINKAGE COHORT STUDY}

${ }^{1} \mathrm{~K}$ Harron*, ${ }^{2} \mathrm{~J}$ Fagg, ${ }^{3} \mathrm{~A}$ Guttmann, ${ }^{4} \mathrm{~J}$ van der Meulen, ${ }^{1} \mathrm{R}$ Gilbert. ${ }^{1}$ Population, Policy and Practice, UCL Great Ormond Street Institute of Child Health, London, UK; ${ }^{2}$ Public Health, Imperial College London, London, UK; ${ }^{3}$ Health System Planning and Evaluation, ICIES, Ontario, Canada; ${ }^{4}$ Health Services Research and Policy, London School of Hygiene and Tropical Medicine, London, UK

\subsection{6/jech-2019-SSMabstracts. 170}

Background Increased support during pregnancy and early childhood is targeted at first time teenage mothers and is recommended for other vulnerable mothers in England. We used population-level healthcare data to assess which mothers and children are most at risk of adverse health outcomes.

Methods We used Hospital Episode Statistics for births to mothers aged 15-44 years in NHS hospitals in England between 2011-2014. We evaluated a number of birth, child and maternal outcomes within 24 months following birth (including birthweight, emergency hospital contacts for children, and subsequent deliveries), according to a range of maternal risk factors: age, parity, neighbourhood deprivation, history of adversity (drug/alcohol abuse, violence, self-harm), or chronic mental or physical illness identified from ICD-10 diagnosis codes in the 5 years prior to delivery.
Results Of the $1,174,688$ births in the study population, $61,370(8 \cdot 7 \%)$ were to first time teenage mothers, 16,675 $(1 \cdot 0 \%)$ were to multiparous teenage mothers, 76,245 (4.4\%) were to mothers with a history of adversity, and 394,388 $(23 \cdot 0 \%)$ were to mothers with a mental health/behavioural chronic condition. The prevalence of risk factors generally decreased with maternal age, but the relationship between risk factors and outcomes varied according to maternal age. Compared with infants born to multiparous mothers aged 25-35 with no risk factors ('low risk' mothers), babies born to primiparous teenage mothers were 206 g lighter (95\% CI 202-211 g) and babies born to multiparous teenage mothers were 206 g lighter (95\% CI 198-215 g). Compared with children born to 'low risk' mothers, an additional $11.9 \%$ (95\% CI $11.5-$ $12.3 \%$ ) of babies born to primiparous teenage mothers and $15 \cdot 6 \%$ (95\% CI $15 \cdot 0-16 \cdot 2 \%)$ born to mothers aged $20-24$ with a history of adversity had an emergency hospital contact. Subsequent deliveries were most common in multiparous teenage mothers $(21.9 \%$ for primiparous and $23.3 \%$ for multiparous teenage mothers, compared with $11 \cdot 1 \%$ for 'low risk' mothers). When adjusting for all maternal risk factors together, mental health conditions were a strong driver of adverse outcomes.

Discussion In addition to primiparous teenage mothers, multiparous teenage mothers and mothers across a range of ages with a history of adversity or mental health condition could benefit from increased support before, during and after birth. Appropriate delivery and targeting of effective interventions for these groups could help improve birth, child and maternal outcomes.

\section{P20 ANALYSIS OF IRISH INQUIRY REPORTS RELATING TO PREGNANCY LOSS SERVICES (2005-2018)}

${ }^{1,2,3} \mathrm{~A}$ Helps*, 'L O'Byrne, ${ }^{2} \mathrm{~S}$ Leitao, ${ }^{1,3} \mathrm{~K}$ O'Donoghue. ${ }^{1}$ Pregnancy Loss Research Group, University College Cork, Cork, Ireland; ${ }^{2}$ National Perinatal Epidemiology Centre, University College Cork, Cork, Ireland; ${ }^{3}$ The Irish Centre for Fetal and Neonatal Translational Research (INFANT), University College Cork, Cork, Ireland

\subsection{6/jech-2019-SSMabstracts. 171}

Background External inquiries related to maternity services are sometimes carried out after negative media reporting of adverse events that have occurred. External inquiries aim to identify issues in the maternity care provided to pregnant women and to make recommendations to improve the standard of care; however, these recommendations are not always implemented. Published literature comparing external reports and assessing their impact on maternity services is limited. Hence, this is the focus of this study.

Methods Ten publically-available national health-service-commissioned inquiry reports published between 2005-2018 relating to perinatal deaths and pregnancy loss services, were identified from national inquiries into the maternity services in Ireland. These were assessed by 2 clinicians, separately, to compare and examine the content and recommendations made in each report.

Quantitative and qualitative data was collected using a specifically designed review tool (based on the Health Service Executive's (HSE) Systems Analysis Review Report Checklist (SARRC)). The findings and recommendations from each report were studied by descriptive thematic analysis outlining emerging themes and issues. 
Results The length of the reports ranged from 11-210 pages (average 86). A definitive purpose for the inquiry was stated in $6 / 10$ reports. Half of the reports explained the inquiry methodology used (including reference to review tools).

The inquiry team was named and multi-disciplinary (MDT) in five reports (4-14 people); four reports described each person's role, but not their responsibilities. It was clearly stated that affected families were involved in four inquiries and relevant clinical staff in four. The inquiry team commented on good aspects of care provided by clinical staff in only four reports.

In the recommendations four main domains were identified: management of information; pregnancy loss care; maternity services governance; workforce staffing and training. The SMART principle (i.e. specific, measurable, achievable, realistic or time-bound) was not consistently applied to the recommendations in any report, thereby reducing the chance of implementation.

Conclusion This was the first structured review of pregnancyloss national inquiry reports, highlighting some of the main issues arising from them. The methodology used during the inquiry process was clearly described in only half of reports. Clearly standardising inquiry processes and highlighting all relevant issues is essential. For this purpose, structured national report checklists can be a useful resource. Additionally, a selected external expert MDT, clinical staff and families should always be involved in the inquiry process. Recommendations made within inquiry reports, can have a profound impact on maternity services if implemented appropriately. This will be explored further in an analysis of all implemented recommendations.

\section{P21 PATHWAYS TO ETHNIC DIFFERENCES IN OBESITY: THE ROLE OF MIGRATION, CULTURE AND SOCIO-ECONOMIC POSITION IN THE UK}

V Higgins*, J Nazroo, M Brown. Cathie Marsh Institute for Social Research, University of Manchester, Manchester, UK

\subsection{6/jech-2019-SSMabstracts. 172}

Background Previous research has identified ethnic differences in abdominal obesity but has not fully explored the pathways that explain these ethnic differences, which may relate to individual and contextual characteristics. This research identifies ethnic differences in waist circumference for eight ethnic groups in England, before and after accounting for a range of individual-level and area-level factors. Three key pathways to obesity are explored: migration, culture, and socio-economic characteristics.

Methods Data come from four years of the Health Survey for England (1998, 1999, 2003 and 2004) and linked area-level data from the 2001 Census. The total sample size is 27,946 . Multi-level modelling methods are used to account for individual-level and area-level factors. The main outcome measure is objectively measured waist circumference. Area-level variables are Index of Multiple Deprivation and Co-ethnic density.

Results The results show that migration to the UK has a strong association with ethnic differences in waist circumference - in particular, waist circumference increases with length of time since migration to the UK. Cultural characteristics and socio-economic characteristics are also associated with ethnic differences in waist circumference, but not to the same extent as migration. The strong association between migration and waist circumference is partly attenuated by cultural characteristics and partly by socio-economic inequality. However, there is still a large association between migrant status and waist circumference that remains unexplained. Area deprivation has an association with ethnic differences in obesity.

Conclusion This research makes an important contribution to the study of ethnic differences in waist circumference in England. It is the first UK study to explore the associations of migration, cultural and socio-economic pathways with ethnic differences in waist circumference for a wide range of ethnic groups, using both individual and contextual data. The resultsillustrate the multi-dimensional nature of ethnic inequalities in obesity and the complex interplay between migration, culture and socio-economic inequality. Policy makers should focus on obesity prevention among child and adult migrants, particularly focussing on length of time since migration, the removal of barriers to educational attainment and employment among migrant populations and the effect of the areas that migrants live, particularly in relation to area deprivation. Obesity is an important factor in many health-related outcomes so the regular tracking of ethnic inequalities in obesity and a clear understanding of the pathways behind these differences is fundamental for improving the health of ethnic minority people.

\section{P22 USING NATIONAL HOSPITALISATION DATA TO EXPLAIN TRENDS IN INPATIENT ADMISSIONS FOR COPD IN IRELAND}

${ }^{1}$ E Hurley ${ }^{*},{ }^{2} \mathrm{~S}$-J Sinnott, ${ }^{3} \mathrm{~J}$ Browne, ${ }^{4} \mathrm{~T} \mathrm{MCDonnell,}{ }^{5} \mathrm{M}$ O'Connor, ${ }^{1} \mathrm{C}$ Normand. ${ }^{1} \mathrm{C}$ entre for Health Policy and Management, Trinity College Dublin, Ireland; ${ }^{2}$ Department of Noncommunicable Disease Epidemiology, London School of Hygiene and Tropical Medicine, UKi ${ }^{3}$ School of Public Health, University College Cork, Cork, Ireland; ${ }^{4}$ School of Medicine, University College Dublin, Ireland; ${ }^{5}$ Department of Public Health, Health Services Executive, Dublin, Ireland

\subsection{6/jech-2019-SSMabstracts.173}

Background Ireland has the highest rates of hospitalisations for Chronic Obstructive Pulmonary Disease (COPD) of all OECD countries $(369 / 100,000$ in 2017 , compared with OECD average of 200/100,000). We analysed national hospitalisation data to investigate the characteristics of these hospitalisations and how they have changed over time to assist in programme planning and identify areas for intervention.

Methods Using Hospital In-Patient Enquiry (HIPE), a national discharge database for episodes of admitted care in acute public hospitals, we undertook a longitudinal review of trends in inpatient hospitalisations for COPD between 2009 and 2017. COPD hospitalisations were identifiable by ICD-10-AM codes J40-J44 and J47. We standardised against two populations: 1) sex-specific rates against the OECD pop and 2) age-sex rates to the Irish population for adults aged 15 years and over

Results The prevalence of overnight hospitalisations increased for females from an age-standardised rate of 367.5/100,000 in 2009 to $393.0 / 100,000$ in 2017, and declined for males 391.6/100,000 to 343.5/100,000. Between 2009 and 2017, age-specific rates in males declined across most age categories, with the decline most pronounced in the 80-84 year age group. In contrast, females saw an increase in rates across the majority of age categories.

In 2017, rates were significantly higher amongst women in each 5 - year age category $\leq 70$ years, apart from the 55-59 